\title{
Variation in the time length for the occurrence of Posterior Capsular Opacification following PMMA versus AcrySof Intraocular lens implants
}

\author{
Raunak Bhavsar ${ }^{1}$, Ritesh Kumar $^{1}$ \\ Department of Biomedical Engineering,SBST, VIT University, Vellore (632014)
}

\begin{abstract}
Aim: To evaluate the significance of the biomaterial composition of the implanted Intra Ocular Lenses, in the occurrence of Posterior Capsular Opacification following a cataract surgery.

Methods: A total of 75 patients who underwent IOL implant surgery were studied. The patients were classified based on the biomaterial of the IOL implanted in the eye following a cataract surgery. Group A consisted of eyes implanted with Polymethyl Methacrylate IOLs and Group B consisted of eyes implanted with AcrySof IOLs. The patients were later treated with YAG laser to clear the Posterior Capsular Opacification at the Ujjain laser center, Ujjain, Madhyapradesh.
\end{abstract}

Results: The incidence of Posterior Capsular Opacification was found to develop earlier in the eyes implanted with PMMA IOLs as compared to Posterior Capsular Opacification occurrence in eyes implanted with AcrySof IOLs.

Conclusion: The incidence of Posterior Capsular Opacification was found to be delayed by the use of AcrySof IOLs when compared to the use of PMMA IOLs.

Index terms:Intraocular lenses, Polymethyl Methacrylate, AcrySof, Posterior Capsular Opacification, Lens epithelial cells, Capsulotomy, Biocompatibility

\section{Introduction}

The occurrence of Posterior Capsular Opacification is a prominent complication observed postoperatively in patients implanted with IOLs following a cataract surgery. The surgery involves removal of the anterior capsular bag, leaving the posterior capsular bag and the anterior capsular edge. Thereafter the lens nucleus and cortex is removed via phacoemulsification, small incision cataract surgery or any other standardized method. The surgery leaves the capsular bag with traces of Lens Epithelial Cells and fibers. The leftover Lens epithelial cells proliferate, undergo abnormal differentiation and migrate from the equator to the center, causing visual axis obscuration and dimness in vision.

The factors that play a role in Posterior Capsular Opacification include biomaterial of the IOL, the edge of the IOL and the surgical skills of the surgeon. This study presents a comparison in the significance of the biomaterial of the IOLs and the shape of the IOLs, in the time length variation for the occurrence of Posterior Capsular Opacification following a cataract surgery. All the surgeries that have been used for this study were performed by the same surgeon thus eliminating any errors that can be caused by differences in surgical skills.

Posterior Capsular Opacification can be treated by posterior capsule scrapping, which is a surgical method or by Nd: YAG laser capsulotomy, which is a non-surgical method.

Under various studies, AcrySof IOLs have been found to have very low incidence of Posterior Capsular Opacification when compared to Posterior Capsular Opacification incidence in PMMA IOLs. This reduced incidence of Posterior Capsular Opacification in AcrySof IOLs is due to lower migration of lens epithelial cells (LECs) on the posterior capsule and their subsequent regression [1]. AcrySof lenses have presented no signs of regeneration of Posterior Capsular Opacification after the capsulotomy via Nd:YAG laser. The following paper represents a comparative study of the occurrence of Posterior Capsular Opacification following PMMA vs AcrySof IOL implantation.

\section{Materials and methods}

75 patients who underwent cataract surgery and treated for Posterior Capsular Opacification in Ujjain laser center were enrolled in the study.

The 44 candidates in Group A were implanted with PMMA IOLs and the 37 candidates in group B candidates were implanted with AcrySof IOLs. The average time duration after which group A candidates required YAG treatment was found out to be 27.6 months, whereas the average time duration after which group B candidates required YAG treatment was found out to be 36 months. 
The occurrence of Posterior Capsular Opacification was found out to be dependent upon the biomaterial composition of the IOL and the edge of the IOL. The surgeries were performed by the same surgeon thus variation in the surgical skill has not influenced the results.

The biocompatibility of the material of the IOL is assessed on the basis of uveal biocompatibility and capsular biocompatibility. The foreign body response of the eye against the implant is inflammatory and is related to the uveal biocompatibility. AcrySof IOLs possibly produce better results in eyes with pre-existing blood-aqueous barrier damage due to its association with lower giant cell counts. The relationship between the LECs within the capsular bag and the IOL determine the capsular biocompatibility of the IOL. AcrySof IOLs showcase lower presence of fibrosis and negligible membrane growth.

Bioactive materials like AcrySof allow a single lens epithelial cells to bond both to the IOL and posterior capsule. This prevents further epithelial ingrowths and is resistant to Posterior Capsular Opacification when compared to PMMA.

Thus, the superiority of the AcrySof IOLs based on the above mentioned facts causes delayed occurrence of Posterior Capsular Opacification in eyes implanted with AcrySof lenses following cataract surgery and makes it a better choice over PMMA IOLs.

\section{Results \& Discussions}

The research covered 75 subjects who underwent cataract surgery following IOL implantation. The average time length after which patients with PMMA IOL showed signs of Posterior Capsular Opacification was 27.6 months whereas the average time length after which patients with AcrySof IOL showed signs of Posterior Capsular Opacification was 36 months. Thus, based on the observations, AcrySof IOLs must be preferred over PMMA IOLs to delay the occurrence of Posterior Capsular Opacification in case it develops.

Further research on IOLs will result in the development of superior designs and biomaterials that will substantially reduce the occurrence of Posterior Capsular Opacification following IOL implantation.

\section{Acknowledgment}

The authors would like to thank the physicians at the Ujjain Laser Center for providing the data.

\section{References}

[1]. MahtabAlamKhanzada et al .Comparative incidence of Posterior Capsular Opacification in AcrySof and PMMA intraocular lenses.Int J opthalmol, Vol 2, No.2, june 18,2009.

[2]. Posterior Capsule Opacification After Lens Implantation Incidence, Risk Factors and Management Abhay R Vasavada, Shetal M Raj, Gauri D Shah, Mayank A Nanavaty Disclosures Expert Rev Ophthalmol. 2013;8(2):141-149. 